\title{
El uso de la evaluación como instrumento de gestión y gobernanza en la Unión Europea
}

\author{
Eduardo Zapico-Goñi \\ Ministerio de Economía y Hacienda. España \\ Ezapico@telefonica.net
}

\begin{abstract}
Resumen
La utilidad potencial de la evaluación parece indiscutible, pero cabe la duda sobre el uso real de la información evaluativa. Su relevancia depende de la coherencia de la evaluación con el modelo de dirección y su contexto. En 1996, la evaluación europea se planteó en relación con los procesos de gestión presupuestaria por resultados. Años después, la iniciativa de Gobernanza Europea identificó la evaluación como un instrumento esencial de apertura, participación, coherencia, responsabilidad y eficacia de las políticas comunitaria. Esta iniciativa se justificó apelando no solo al déficit democrático sino además al déficit de capacidad de coordinación europea para dirigir. También existe un "déficit de evaluación" en la UE por el débil desarrollo de una aproximación a la evaluación que responda adecuadamente al contexto. La relevancia de la evaluación en la UE aumentaría significativamente si el esfuerzo de evaluación se reorientara hacia una mayor coherencia con el modelo de dirección y la complejidad del contexto.
\end{abstract}

Palabras clave

Evaluación en la UE, gestión por resultados, gobernanza, incertidumbre, uso de la evaluación.

\section{Using Evaluation for European Management and Governance}

\begin{abstract}
There is no doubt about the potential of evaluation for decision making. Yet, it is not clear what the real evaluation information use is. Its relevance depends on the relation of evaluation approach to the decision making model and their coherence with their context. In 1996, EU evaluation was first oriented towards efficient expenditure management. Years later, the European Governance acknowledged evaluation as a main instrument for transparency, participation, coherence, accountability and effectiveness of EU policy making. EG was justified not only to cover the democratic deficit but also a coordination deficit. Furthermore, an evaluation deficit has been identified due to the weak development of an evaluation approach able to tackle the complexity of the European context. The relevance of evaluation and its use in the EU would increase significantly if evaluation efforts were reallocated towards a decision model coherent with complexity.
\end{abstract}

Key words

EU evaluation, management by results, Governance, uncertainty, use of evaluation. 


\section{INTRODUCCIÓN}

En estos momentos de crisis y grandes transformaciones socio-económicas son pocas las iniciativas comunitarias que se salvan de un cuestionamiento y debate sobre su oportunidad y el valor que aportan. En una reciente comunicación de la Comisión Europea, el presidente Barroso resalta la importante contribución que se espera de la evaluación para maximizar la calidad y la eficacia de las propuestas comunitarias y mostrar que la UE trabaja con responsabilidad y profesionalidad. Según esta comunicación todas las propuestas significativas de revisión de medidas existentes o de nuevas medidas deberán basarse en una evaluación del marco de la política publica correspondiente (European Commission, 2010). Sin embargo, aunque la situación hace evidente la necesidad de producir conocimiento evaluativo, no acaba de despejarse la duda sobre la utilización práctica de la evaluación de programas públicos para el cambio en las políticas públicas y la reasignación de recursos (OCDE, 2007).

Después de unas dos décadas de esfuerzos, la evaluación de políticas públicas en la Unión Europa parece estar funcionando tanto desde el punto de vista de la oferta como de la demanda. En referencia a experiencias de principios de esta década, algunos estudios comparados mostraban que la Comisión había conseguido establecer la evaluación y crear una cultura evaluativa muy influyente en el crecimiento de su practica en la UE y en los Estados miembros (EEMM) (Summa y Toulemonde, 2002; Ernest y Young, 2008). Actualmente, el esfuerzo de evaluación es amplio y coordinado y parece disponer de un buen potencial de desarrollo. Desde el año 2007, la Comisión ha puesto en marcha varias iniciativas orientadas a mejorar la calidad de la práctica evaluativa y potenciar un mayor uso de sus resultados en la elaboración de políticas europeas.

La utilidad de la evaluación en la UE se ha buscado principalmente en el marco de la gestión por resultados, inspirada en el NPM (New Public Management), con el fin de fomentar la competición y maximizar así la utilidad de cada organización o cada iniciativa. Sin embargo, la validez y coherencia de este modelo con la realidad en la que se desarrollan las políticas europeas están cada vez más cuestionadas. Las políticas europeas, como iniciativas de reforma, se desarrollan en contextos desestructurados y exigen una aproximación adaptativa y participativa (Berman, 1980). El contexto político y socioeconómico en el que están inmersas las políticas europeas es cada vez más complejo y las opciones de decisión y sus resultados, previstos o reales, son inciertas. En general, este está caracterizado por la pluralidad y

ô diversidad de intereses; cambio sociopolítico y tecnológico; interdependencia entre sectores; incertidumbre sobre las relaciones causales de la intervención; etc. (ver introducción del presente número). Basándose en el modelo de gobernanza, la respuesta de la UE a la creciente complejidad del contexto ha sido iniciar un proceso de apertura, participación y colaboración en el ciclo de las políticas europeas orientado a maximizar la utilidad colectiva que exige una aproximación distinta a la 102 evaluación. 
Ambos modelos intentan responder a las demandas de la sociedad y para ello cuentan con la evaluación como un instrumento, entre otros, de decisión y dirección estratégica. Aunque hay una gran diferencia entre el modelo gerencial (gestión por resultados) y el de Gobernanza ("gestión" de redes), esto no significa que la evaluación no pueda ser compatible con ambos modelos. Existe un consenso generalizado sobre la utilidad potencial de la evaluación para dirigir Europa, pero cabe la duda sobre la utilidad y el tipo de uso dado en la práctica a la información evaluativa. Tanto la utilización de la información evaluativa como su relevancia dependen de la relación y adaptación de la evaluación a estos dos modelos de decisión y de su coherencia con el contexto de las políticas comunitarias.

En este artículo se analiza la situación en la que se encuentra hoy la evaluación en la UE y se presentan evidencias de que el sistema de evaluación funciona y produce información evaluativa que es utilizada en los procesos de toma de decisiones. El objetivo final es examinar el tipo de uso dado a la evaluación, ilustrándolo con experiencias de buenas prácticas, y analizar su coherencia con el contexto. Se pretende mostrar que el sistema de evaluación de la Unión Europea está bien arraigado y sus recomendaciones se utilizan, aunque de manera limitada, tanto bajo la perspectiva de la gestión por resultados como de la gobernanza. Así mismo, se propone la necesidad de una mayor coherencia de los modelos de dirección y evaluación con el contexto de las políticas europeas. La relevancia del beneficio aportado por la evaluación y su uso para la dirección de la UE aumentaría de manera significativa, si los recursos y esfuerzos de evaluación de la Comisión se reorientaran en busca de mejorar esta coherencia. El análisis se basa en la información recogida de publicaciones previas, documentos oficiales, evaluaciones y documentos de trabajo de la UE.

En las siguientes secciones, en primer lugar, se describe el origen y la evolución de la evaluación en la UE y el marco institucional del sistema de evaluación. Posteriormente se explora la política de evaluación y su enfoque. Finalmente, se analiza y valoran los avances conseguidos, los principales obstáculos encontrados y los tipos de uso de la evaluación.

\section{ORIGEN Y EVOLUCIÓN DEL SISTEMA DE EVALUACIÓN EN LA UE: NAVEGANDO ENTRE LA GESTIÓN POR RESULTADOS Y LA GOBERNANZA}

Dejando a un lado, por motivos de espacio, las experiencias sectoriales de algunas Direcciones Generales (DG) (por ejemplo: Ayuda Exterior, Política Regional e Investigación y Desarrollo), el origen y razón de un sistema global de evaluación en la UE están relacionados con dos reformas de amplio alcance basadas en los modelos de gestión y presupuestación por resultados y de "Gobernanza", que respondían a los dos déficits que preocupan a la UE, el financiero y el democrático. En un principio, la evaluación en la UE se planteó con una clara función técnico-organiza- 
tiva relacionada con los procesos de gestión y particularmente de gestión del gasto. La preocupación inicial fue valorar la eficacia y eficiencia de las iniciativas comunitarias con consecuencias significativas en el presupuesto de la UE. Casi en paralelo a esta aproximación se han realizado importantes esfuerzos de evaluación en el marco de la iniciativa de la Gobernanza Europea. La evolución de la evaluación ha ido prestando más atención a otro tipo de medidas, Ilamadas de no-gasto (por ejemplo: legislativas, informativas, de coordinación, etc.) e incluyendo otro tipo de criterios de valor como la relevancia, coherencia, sostenibilidad, transparencia, participación, etc. de las políticas comunitarias. Específicamente, la introducción y mejora del sistema de evaluación en la UE han ido estrechamente unidas a las siguientes reformas.

\subsection{La reforma SEM (Sound and Efficient Management) 2000}

Desde hace varias décadas los organismos internacionales tales como la Organización para la Cooperación y Desarrollo Económico (OCDE) y el Fondo Monetario Internacional (FMI), vienen proponiendo e impulsado reformas en la gestión del gasto público orientadas a introducir el presupuesto por resultados y aplicar los criterios de economía, eficiencia y eficacia en el marco de la Nueva Gestión Pública (OCDE 1996, 2002, 2007; IMF, 2005). El modelo racional de gestión y presupuesto por resultados es bien conocido. A través de la competición se incentiva un comportamiento de decisión que trata de maximizar beneficios a nivel departamental, sin prestar la suficiente atención a la utilidad colectiva y bajo supuestos de lógicas de intervención lineales (input, action, output, outcome). Se supone que los procesos de decisión se llevan a cabo en un entorno simple o estructurado (pocos participantes y con autonomía de acción, clara delimitación de responsabilidades, baja resistencia al cambio, ausencia de conflictos de valores o enfrentamiento de intereses, estabilidad y validez comprobada de las políticas). Son múltiples los estudios realizados sobre el potencial y las dificultades de la integración y uso de la información sobre resultados en el presupuesto (Shick, 2001; Pollitt y Bouckaert, 2000; Diamond, 2003; Mayne 2007). En múltiples estudios se proponen vías para mejorar la asignación de los recursos en función de la evaluación de programas dentro y más allá del paradigma de la New Public Management (NPM) (Gray, Jenkins y Segsworth, 1993; Mayne y Zapico, 1995; Subirats, 2004; Robinson, 2007; Radej, Golobic y Cernic, 2010).

Con este modelo de gestión se corresponde la visión tradicional de la Comisión Europea como entidad responsable de la preparación de medidas legales y presupuestarias y de su gestión directa o del seguimiento y control de las actuaciones de las Agencias europeas y de los Estados miembros como ejecutores de las medidas europeas. Bajo esta perspectiva la evaluación de las políticas públicas se centra en el análisis del grado de cumplimiento de los objetivos plasmados en las medidas europeas y en el estudio de las relaciones entre recursos, operaciones y 104 resultados. 
En línea con este modelo, la Comisión Europea inicia a finales del siglo pasado su reforma en la gestión presupuestaria como respuesta al grave problema del aumento del gasto en la Unión Europea causado en parte por el aumento de funciones y nuevas competencias como las políticas en investigación y desarrollo, educación, medio ambiente, sociedad de la información, etc., y la creciente presión por una mayor disciplina fiscal (condiciones o criterios de Maastricht) exigida a los Estados miembros y consecuentemente a la Comisión Europea.

La evaluación en la UE se origina por tanto como un instrumento de gestión por resultados de los programas presupuestarios y de reasignación de los recursos. El gasto se estructura en torno a grandes partidas llamadas "Actividades presupuestarias" que en muchos casos son políticas u organizaciones europeas (por ejemplo: Fondos Estructurales, programas marco de actuación en distintos sectores, agencias reguladoras y ejecutivas, etc.). El número, normalmente pequeño, de estas grandes políticas o líneas de trabajo, o agencias dependientes, de cada Dirección General se presenta de manera codificada en el documento presupuestario y debe ser objeto de seguimiento y evaluación (European Commission, 2000). También se presentan en el presupuesto "Actividades sin Línea Presupuestaria" (Activity Without Budget Line), sin consecuencias directas de gasto que pueden ser objeto de evaluación, pero no obligatoriamente. El Reglamento Financiero de la UE formalizó (2002) la obligación de evaluar y utilizar los resultados de la evaluación para justificar las variaciones de los créditos presupuestarios solicitados por las Direcciones Generales. Por lo tanto, legalmente, el objeto de la evaluación son grandes "Actividades" o políticas identificadas y codificadas en el presupuesto, sin embargo en la practica el objeto de las evaluaciones suelen ser iniciativas o medidas de menor dimensión cuyos resultados a menudo son difíciles de relacionar con las grandes partidas o "Actividades" presupuestarias, por ley objeto obligado de evaluación.

Paralelamente la política de evaluación se ha ido ampliando y desarrollando en el marco de otras iniciativas de reforma en la UE.

\subsection{Las Comunicaciones sobre Evaluación $(1996,2000,2007 a)$ y sobre funciones relacionadas con la Gestión por Resultados $(2001,2002)$}

Las tres Comunicaciones sobre evaluación marcan con claridad la evolución del sistema de evaluación en la Comisión Europea hasta finales del 2008. En el 2009 se inicia una transición del sistema de evaluación como veremos más adelante. La creación de un sistema global de evaluación para la UE en 1996 no partió de cero, dado que antes de esa fecha ya se disponía de experiencia y capacidad de evaluación en algunas Direcciones Generales (Agricultura, Ayuda y Cooperación Internacional, Política Regional y otras). En 1996, sin embargo, con la primera comunicación sobre evaluación, se crea una política global común para toda la Comisión Europea, que oriente la tarea de las Direcciones Generales sin experiencia en evaluación y que les facilite 
aprender de los conocimientos de las Direcciones Generales que tenían ya una cierta trayectoria en evaluación.

Con el cambio de siglo se abre una nueva fase en el desarrollo de la evaluación en la UE en la que se asocia la evaluación al intento de que los resultados y las evidencias encontradas sean útiles para la toma de decisiones y la mejora de sus políticas. Se da otro impulso a la evaluación con una nueva Comunicación (2000) que anima a que los resultados de las evaluaciones se tengan en cuenta a lo largo del ciclo de políticas públicas. Simultáneamente, se realizan una serie de comunicaciones sobre gestión por resultados, Activity Based Management (ABM) (European Commission, 2001), y la evaluación de impacto, Impact Assessment (IA) (European Commission, 2002). Menos la comunicación del Activity Based Budget (ABB), liderada por la Dirección General de Presupuestos, el resto se han desarrollado desde la Secretaría General (SG) de la Comisión. En todas ellas hay claros y explícitos mensajes sobre el rol instrumental de la evaluación en sintonía con estas técnicas de gestión.

En el año 2007 se aprobó una nueva comunicación que pretende elevar la utilidad de la evaluación al máximo nivel estratégico de decisión. Se busca que la evaluación no se utilice sólo a nivel de medidas sectorial y de gestión operativa, sino que también se enlacen los resultados de la evaluación con las grandes políticas y prioridades de la Comisión. Se busca que de la evaluación de las medidas o programas de acción surja información útil para identificar en qué grado y cómo éstas contribuyen a impulsar o alcanzar los objetivos de cada una de las grandes políticas sectoriales y prioridades de la Comisión. La evaluación se entiende como una respuesta a las necesidades de información estratégica de la Comisión y supone un avance sobre las dos fases anteriores.

Finalmente, desde el 15 de enero de 2009 la unidad (coordinadora del sistema) de evaluación ha sido transferida de la Dirección General de Presupuesto a la Secretaría General (SG) de la Comisión Europea. Este cambio supone el inicio de una nueva etapa en la producción y uso de la información evaluativa que, sin dejar de prestar atención a la faceta presupuestaria, pone un mayor énfasis en el ciclo de gestión (ABM) y en complementar la evaluación ex ante o valoración de medidas legislativas (IA) con la evaluación (interina o ex post). No obstante, debido a que el 2009 ha sido un año transición en la UE, por el momento no ha habido cambios de relevancia en el de evaluación.

\subsection{El Libro Blanco sobre la Gobernanza en la UE (LBG)}

Desde hace dos décadas se viene desarrollando el estudio de los procesos de dirección de la UE en el marco de la gobernanza (Majone, 1991; Scharpf, 2002; Metcalfe, 1996, 2001, 2004; Borrás y Jacobsson, 2004; Stame, 2008; Jordana, Mota y Noferini, 2009). La visión de la Comisión europea como una organización en red cambia de una manera ra-

106 dical el modelo no solo de decisión sino también de evaluación (por ejemplo: del objeto 
evaluado, de los criterios de valor) y del uso de sus resultados. El rol de la Comisión como un actor central que debería liderar la creación y funcionamiento de las redes y regimenes de políticas comunitarias (Metcalfe, 1996) es de gran relevancia. Se han identificado y analizado roles de la Comisión como coordinador e intermediario central de las redes comunitarias (Borrás, 2009) que van más allá de la preparación de medidas legales y del seguimiento de su implantación. Los resultados de las políticas de la UE se pueden y deben enjuiciar también en el marco del funcionamiento de estas redes.

En el primer artículo de este monográfico, L. Aguilar y M. Bustelo definen en general la gobernanza como la actividad de gobierno o dirección de la sociedad orientada a satisfacer unas preferencias sociales definidas entre el gobierno y la sociedad, y realizadas con unas acciones, recursos, instrumentos y formas de organización consensuadas. En este marco, el gobierno dirige a través de redes de colaboración con los actores económicos y sociales. El rol del gobierno no es tanto dirigir y ejecutar las políticas públicas, sino fomentar la creación y fortalecimiento de redes de trabajo e incentivar procesos de participación, asociación, coordinación y resolución de conflictos, en las que se comparte la responsabilidad por el buen funcionamiento y desarrollo de la gobernanza.

El Libro Blanco de Gobernanza de la UE (European Commission, 2001b) identifica la evaluación como uno de los instrumentos esenciales para la gobernanza europea. La iniciativa de gobernanza se ha orientado a simplificar y mejorar la regulación y la preparación de las políticas comunitarias basándose, entre otros instrumentos, en la evaluación ex ante (IA). Además se abre la posibilidad de utilizar instrumentos alternativos a los regulatorios (por ejemplo: Open Method Coordination). Con esta reforma, la UE se propuso que los procesos de decisión se realizaran bajo los principios de apertura, participación, coherencia, responsabilidad y eficacia. Esta iniciativa elevó cualitativamente el potencial de la evaluación como instrumento de decisión pero con una perspectiva muy distinta a la de sus orígenes.

La apertura y participación en la conformación de las políticas Europeas puede ser beneficiada por las posibilidades que ofrecen ciertos métodos y aproximaciones a la evaluación (por ejemplo: análisis de redes, evaluación participativa). El LBG propuso la apertura de las políticas públicas de la UE para asociar un mayor número de actores en su programación, ejecución y control, que se traduciría en una mayor transparencia, participación y responsabilización de todos los stakeholders. De esta manera, los ciudadanos podrían comprobar cómo los Estados miembros, actuando de manera conjunta en el marco de la Unión Europea, son capaces de responder más eficazmente a sus necesidades y preocupaciones. Esto requiere un esfuerzo no solo de la Comisión Europea sino de las demás instituciones europeas (Consejo y Parlamento), de los Estados miembros y de otros niveles de gobierno, del mercado y de la sociedad civil.

Por lo tanto, la evaluación se considera un instrumento para diseñar y mejorar la legislación y las políticas europeas en base a criterios, conocimientos y esfuerzos compartidos. Con este fin se plantea mejorar la calidad de la evaluación con propuestas ta- 
les como: a) reforzar la cooperación interinstitucional (Parlamento Europeo - Consejo de Ministros - Comisión Europea) para la evaluación y desarrollar una colaboración (evaluation partnerships) más estrecha con los Estados Miembros y la sociedad; b) aumentar la capacidad de evaluación de la Comisión y los Estados miembros; c) desarrollar instrumentos y procedimientos participativos para evaluar la acción legislativa y presupuestaria de la UE; e) mejorar sistemas de seguimiento y disponibilidad de datos empíricos en los Estados miembros; f) integrar la evaluación en las funciones de planificación y presupuesto; aumentar la transparencia y difusión proactiva de los resultados de la evaluación; g) construir una cultura de resultados que conlleve una toma de decisiones basada en el análisis más que en concesiones ad hoc (European Commission, 2001c).

\section{MARCO LEGAL E INSTITUCIONAL DE LA EVALUACIÓN}

En la UE hay una clara obligación legal de evaluar, reflejada explícitamente en su Reglamento Financiero (European Comission, 2002a) que exige la evaluación de toda intervención que ocasione un gasto significativo en el presupuesto de la UE. Particularmente y de manera muy ambiciosa, el artículo 33 señala que el resultado de la evaluación se debe utilizar para justificar las solicitudes y las variaciones de crédito que se presenten en el proceso presupuestario. También los reglamentos específicos de algunos sectores o Direcciones Generales incluyen la obligación y condiciones de evaluar no sólo a la Comisión sino también a los Estados miembros (por ejemplo: Fondos Estructurales). Otras normas secundarias, las Comunicaciones de Evaluación (1996, 2000, y 2007) y de Impact Assessment (European Commission, 2002b) y los Estándares de Evaluación y Buenas Prácticas (European Commission, 2007) completan el marco normativo de la evaluación en la UE. Este marco, en general, está bien diseñado, es comprehensivo y se ha ido adaptando mutuamente con la evolución de la práctica evaluativa.

La configuración institucional de la evaluación es compleja pero, en general, permite un funcionamiento adecuado del sistema de evaluación, tanto desde la perspectiva de la demanda como de la oferta de evaluación. El sistema de evaluación se localiza en la Comisión Europea. Se trata de un sistema interno de evaluación que hasta el 2009 se ha coordinado desde la Dirección General de Presupuestos. Actualmente se coordina desde la Secretaría General. El Parlamento Europeo (PE) y el Consejo de Ministros (CM) no disponen de servicios de evaluación. En el Parlamento Europeo se viene reclamando el desarrollo de su capacidad de evaluación. De hecho tanto el PE como el Consejo de Ministros ejercen una presión continua para que mejore la calidad y aumente el uso de la evaluación. El Consejo de Ministros y el Parlamento requieren a la Comisión información específica sobre el estado o la calidad de la información evaluativa incorporada en los planes de actividades y la documentación presupuestaria. Las nuevas formas de colaboración y co-decisión entre las Instituciones europeas suponen una oportunidad para que estas dos instituciones aumenten su papel en la evaluación de las políticas europeas. Ambos tienen competencias para llevar a cabo evaluaciones, parti108 cularmente en los Impact Assessment (IA), aunque por el momento prácticamente no 
se hayan ejercido. Recientemente se ha propuesto que el PE y el Consejo de Ministros realicen evaluaciones ex ante de sus propias enmiendas (Stern, 2009).

Además de su representación en el Consejo de Ministros, los Estados miembros son también importantes actores del sistema de evaluación Europea. En algunas políticas europeas (por ejemplo: Política Regional, Empleo, Ampliación) la responsabilidad de evaluar reside en gran medida en los gobiernos nacionales.

El Tribunal de Cuentas Europeo (TCE) no sólo utiliza los informes de evaluación para la realización de sus trabajos de auditoria, sino que además sus auditorias van más allá de la mera revisión financiera y aplican métodos y criterios parecidos a los de la evaluación. El TCE ha auditado el propio sistema de evaluación de la Comisión Europea y ha proporcionado una información muy valiosa para mejorar su funcionamiento (ECA, 2006).

El cuadro siguiente recoge de manera informal una imagen global de las principales instituciones comunitarias y foros más activos o interesados en la evaluación: el Parlamento Europeo, el Consejo de Ministros, los Estados miembros y la Comisión Europea con sus "Funciones de Evaluación" y la unidad central de coordinación. Todas estas instituciones se sitúan en torno a varios foros de audiencia y consulta donde se demanda conocimiento evaluativo para el debate y la toma de decisiones (ver Cuadro 1 y acrónimos).

\section{CUADRO 1}

\section{Red Institucional de Evaluación*}

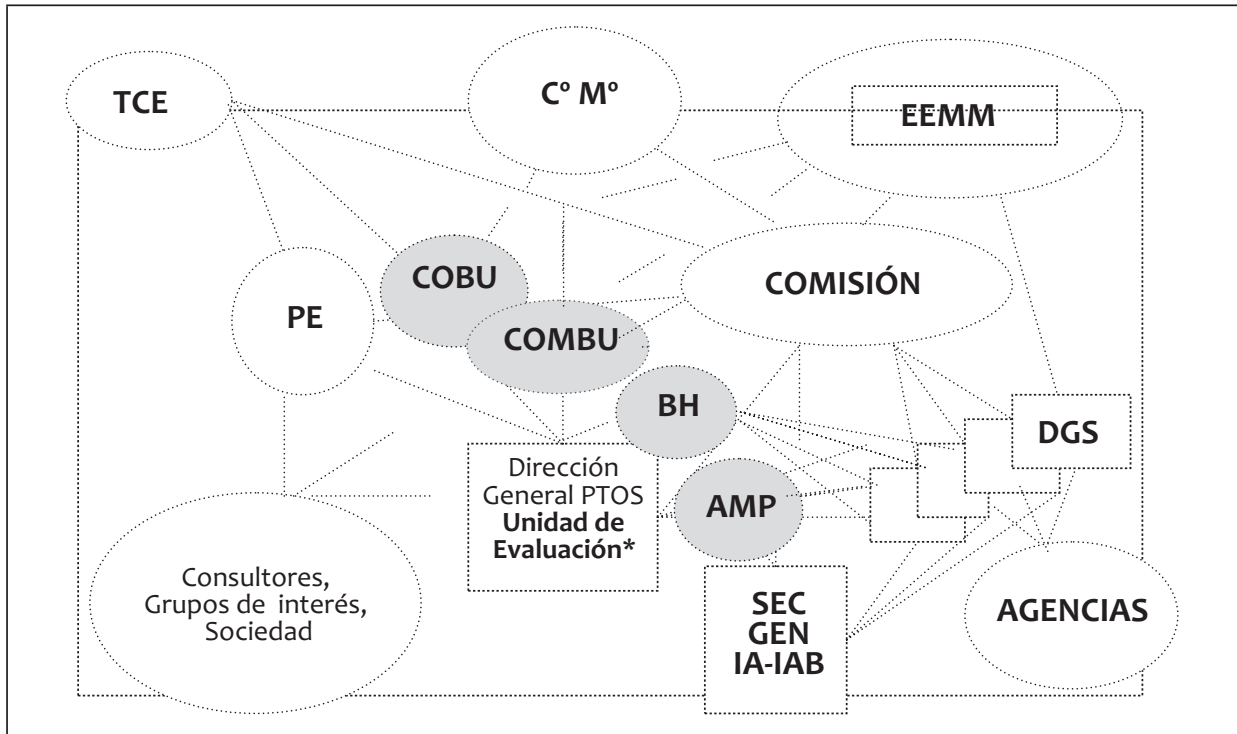

* A partir del 15/01/09 la Unidad de Evaluación (hasta esta fecha situada en la Dirección General de Presupuestos que pertenece a la Secretaría General).

Fuente: Elaboración Propia (ver significado de acrónimos que sigue a continuación). 
Significado de los acrónimos presentados en el Cuadro 1

- SEC. GEN.: Secretaría General de la Comisión.

- BH: Audiencias Presupuestarias (Budget Hearings).

- EE MM: Estados miembros.

- DGS: Direcciones Generales.

- Dirección General Ptos: Dirección General de Presupuestos.

- $\mathrm{C}^{\circ} \mathrm{M}^{\circ}$ : Consejo de Ministros.

- COMBU: Comité de Presupuestos del Consejo de Ministros.

- COBU: Comité de Presupuestos del Parlamento Europeo.

- PE: Parlamento Europeo.

- Dirección General: Direcciones Generales.

- EF: Unidades de evaluación (Evaluation Functions).

- FMU: Unidades de Gestión Financieras (Financial Management Units).

- AMP: Planes Anuales de Gestión (Annual Management Plans).

- TCE: Tribunal de Cuentas Europeo (European Court of Auditors).

- IA: Valoraciones de Impacto (Impact Assessment).

Los cuatro círculos pequeños ensombrecidos en la imagen representan sendos foros de debate en los que se demanda y se presenta información evaluativa: a) las reuniones de planificación-programación, donde se analizan los Planes Anuales de Gestión (AMP, por sus siglas en inglés) entre las Direcciones Generales y la Secretaría General de la Comisión; las Audiencias Presupuestarias o Budget Hearings (BH), donde tienen lugar las discusiones bilaterales entre las Direcciones Generales de la Comisión y la Dirección General de Presupuestos para asignar los recursos en cada ejercicio; y los Comités Presupuestarios del Consejo de Ministros (COMBU) y del Parlamento Europeo (COBU).

En estas reuniones y comités se demanda y se presenta información sobre los resultados de las evaluaciones, incluidos los IA, para justificar la programación de actividades y apoyar las demandas de recursos financieros y de personal. No obstante, en general, tanto las decisiones de programación como las de asignación de recursos se siguen basando en procesos de negociación en torno a intereses y conveniencias políticas, buscándose a menudo compromisos rápidos no necesariamente basados en los resultados de los estudios y análisis realizados por la Comisión. Durante la segunda mitad de esta década se han realizado, en el marco de las reuniones de los comités presupuestarios del Consejo de Ministros (COMBU) y del Parlamento Europeo (COBU), estudios anuales sobre la mejora de la información evaluativa, seguidos de los correspondientes requerimientos a la Comisión para avanzar de acuerdo a sus recomendaciones (Consejo de Ministros UE, 2008; European Parlament, 2008).

La red institucional de la evaluación en la UE esta bien diseñada, las competencias están bien distribuidas, los roles de producción y uso de la evaluación están convenientemente definidos y las relaciones entre los actores están claramente definidas. Sin embargo, existen ciertas limitaciones y obstáculos a la hora de intentar utilizar los resultados de la evaluación para la toma de decisiones, como veremos en 110 las siguientes secciones. 
El sistema de evaluación tiene tres importantes características organizacionales: es descentralizado, tiene capacidad de aprendizaje y funciona de manera abierta y en red. En primer lugar, en relación a su naturaleza descentralizada, cada Dirección General realiza su actividad evaluadora de manera autónoma. Dentro de cada Dirección General existen unas unidades o departamentos denominados "Funciones de Evaluación” (FE) responsables de planificar, coordinar y controlar las evaluaciones que se desarrollen en su Dirección General. Las FE se coordinan desde la unidad central de evaluación de la Secretaría General mediante una Red de Evaluación. En colaboración con las “Funciones de evaluación”, la unidad central de evaluación es responsable de preparar normas y estándares de evaluación, promover buenas prácticas, apoyar subsidiariamente a las funciones de evaluación que lo necesiten, verificar la incorporación de información evaluativa en las fases de planificación y presupuestación, recopilar y sintetizar los resultados de las evaluaciones llevadas a cabo en todas las Direcciones Generales.

La Secretaría General también coordina un sistema descentralizado de evaluación o valoraciones de impacto (Impact Assessment) de naturaleza prospectiva. El IA se introduce en la Comisión en el contexto de las Estrategias de Lisboa y de DesarroIlo Sostenible y el debate sobre Gobernanza. Los IA están orientados a contribuir a la eficacia, transparencia, calidad y coherencia del desarrollo participativo de las políticas europeas. Para la Comisión los IA son evaluaciones ex ante de los efectos estimados, directos e inducidos, de las propuestas legislativas o medidas de actuación de la UE para identificar y analizar las posibles opciones o líneas de reforma. Los IA van más allá del examen de las consecuencias que la regulación pueda tener en los costes administrativos de las empresas. Se analizan de manera integrada las alternativas de decisión y los posibles "trade-offs" o contrapesos de sus efectos desde una triple perspectiva: económica, social y medioambiental (European Commission, 2002b).

Los IA utilizan una metodología análoga a los de la evaluación (retrospectiva). Ambas aproximaciones son complementarias. Los IA deben tener en cuenta los resultados de evaluaciones pasadas y presentar un marco (principales objetivos e indicadores de éxito, calendario y responsables) para la evaluación in terim y ex post de las medidas o iniciativas estudiadas. La evaluación se realiza en referencia al marco presentado en el IA. La gestión de los IA y el control de su calidad siguen procedimientos diferentes al de la evaluación. Por ejemplo, la calidad de los IA está sometida a la revisión del Consejo de Evaluación de impacto (Impact Assessment Board, IAB) que depende directamente del Presidente de la Comisión Europea, de manera independiente de las Direcciones Generales, mientras que la revisión de la calidad de las evaluaciones ex post sigue un proceso formalizado y estandarizado pero de auto valoración. El trabajo realizado por la IAB está suponiendo una mejora sustancial de los IA (Robertson, 2008) en relación a un decepcionante arranque con calidad decreciente entre el 2002 y 2005 (Renda, 2006).

En segundo lugar, el sistema de evaluación de la UE dispone de instrumentos eficaces para el aprendizaje. Se utilizan sistemas de seguimiento y control de las eva- 
luaciones; por ejemplo los Grupos de Dirección y Seguimiento (Steering Groups) de cada evaluación realizan un trabajo de gran utilidad en este sentido. En estos grupos participan los departamentos involucrados o relacionados con la iniciativa evaluada, la Función de Evaluación, expertos independientes y grupos de interés afectados por la iniciativa. Su funcionamiento permite un intercambio de información y conocimiento esencial para la preparación y desarrollo de la evaluación en coherencia con los términos de referencia y la difusión y utilidad de sus resultados.

Los marcos de calidad de los informes de evaluación que no sólo califican ciertas características de los informes (por ejemplo: pertinencia del diseño, credibilidad de los datos, solidez del análisis, validez y coherencia de resultados y conclusiones, utilidad de las recomendaciones), sino que además permiten captar y acumular información y desarrollar conocimiento para mejorar la capacidad de evaluar (European Comission, 2008c; European Comission, 2009).

Además, las Direcciones Generales disponen de procedimientos de actuación específicos de feedback y seguimiento de la aplicación de los resultados de la evaluación. Estos procedimientos están pensados para facilitar y comprobar el uso de los resultados de la evaluación. Pero además permiten que, en su caso, los responsables de la implantación de las recomendaciones puedan presentar su opinión y proporcionar información sobre el proceso y los resultados de su implantación. Uno de los casos más notorios es el de la Fiche Contradictoire (FC) de la Dirección General de Ayuda Internacional. La FC es un mecanismo formalizado y estandarizado para el seguimiento del uso de la evaluación. Esta debe ser cumplimentada después de toda evaluación. La ficha tiene tres columnas: la primera para identificar la recomendación, la segunda para incorporar las respuestas de la unidad responsable y la tercera, a cumplimentar un año tras la finalización de la evaluación, para mostrar las acciones tomadas por los servicios responsables. A través de estas fichas la unidad de evaluación recibe información y feedback de las unidades responsables de aplicar las recomendaciones. Estas FC han proporcionado una información importante para la gestión de mejoras a nivel operativo o sectorial y han ejercido una cierta presión y motivación para las unidades responsables de implantar las recomendaciones. En la práctica han sido además un buen mecanismo de defensa de estas frente a las críticas externas a la Comisión por no llevar a cabo ciertas recomendaciones.

Por otro lado, el aprendizaje y desarrollo global del sistema de evaluación se ha llevado a cabo en base a estudios en profundidad y sistemáticos sobre el mismo, como por ejemplo, el realizado para la Dirección General de Presupuestos (EPEC, 2005) sobre el uso de los resultados de la evaluación en la Comisión y el realizado por el TCE sobre el funcionamiento del sistema de evaluación (ECA, 2006).

Por último, la organización y funcionamiento del sistema de evaluación de la UE es en red y abierto. Una de las principales características del sistema de producción de evaluación en la UE es su significativa dependencia de los recursos y capacidad de 112 evaluación fuera de la Comisión tanto en el sector publico (p.ej. las redes de evalua- 
ción en los Estados Miembros en políticas tales como la Regional, Investigación, Empleo) como en el sector privado (por ejemplo, consorcios de evaluación, grupos de consultores, académicos). Hasta el 2009, aproximadamente un $70 \%$ del esfuerzo de evaluación se contrató con empresas de consultoría, organizaciones especializadas en cada sector, universidades, etc. Estos consultores externos llevan a cabo evaluaciones de gran dimensión y alcance que están fuera de la capacidad interna de la Comisión.

Los sistemas de evaluación de algunas Direcciones Generales cuya gestión es compartida o descentralizada dependen de la capacidad de evaluación en los Estados miembros. Como se ha demostrado hace tiempo los esfuerzos de evaluación de las iniciativas europeas, particularmente de la Política Regional y la de Empleo, se realizan dentro y fuera de la Comisión, a múltiples niveles de gobierno (Rieper, 1997). Estas Direcciones Generales disponen de redes de evaluación (externas) en las que participan los Estados miembros de la UE. Estas redes están bien diseñadas y funcionan con eficacia. Los departamentos o entes estatales que representan a cada país son distintos según la red o el país de que se trate. Se reúnen de manera periódica para debatir y desarrollar normativa, métodos y prácticas de evaluación. Su rol es esencial para la participación de los Estados miembros en la configuración del marco de evaluación, la transparencia sobre los resultados y el reparto de responsabilidades. La labor de estas redes es esencial para el desarrollo del sistema de evaluación de la UE.

La red interna de evaluación de la Comisión (Comission Evaluation Network) es una red administrativa. Está compuesta por los representantes de las funciones de evaluación de cada Dirección General. El pleno de sus miembros se reunía unas 5 ocasiones al año, hasta diciembre de 2008 bajo la coordinación de la unidad de evaluación de la Dirección General de Presupuestos, desde el 2009 esta red se coordina desde la Secretaría General. Las reuniones se orientan principalmente para intercambiar información, fomentar la coordinación y coherencia de los esfuerzos de evaluación y el aprendizaje mutuo. La red fomenta el desarrollo y conocimiento evaluativo colectivo de las funciones de Evaluación. Se organizan reuniones de grupos de trabajo para el desarrollo de metodologías de evaluación y la resolución de problemas de funcionamiento del sistema. Cada año se prepara un plan de actividades de grupos de trabajo, seminarios, cursos de formación, etc. En el 2009 se reunió en una sola ocasión para intercambiar opiniones sobre nuevas líneas a seguir en la práctica de la evaluación tras la transferencia de la unidad de evaluación desde la Dirección General de Presupuestos a la Secretaría General. Existe también una red diferenciada de IA (evaluación ex ante) también coordinada desde la Secretaría General, que se reúne con una regularidad y un funcionamiento similar a la red de evaluación. Estas dos redes son esenciales para que el sistema de evaluación funcione y se desarrolle de manera coordinada.

Dentro de las Direcciones Generales suele haber también redes de evaluación internas en las que participan diversas unidades de la propia Dirección General que 
realizan actividades relacionadas con la evaluación. Estas suelen crearse en las Direcciones Generales con funciones de evaluación pequeñas que descentralizan el seguimiento de las evaluaciones entre los servicios de la Dirección General. Por ejemplo, en la Dirección General de Sanidad y Consumo, la función de evaluación trabaja de manera descentralizada. Cada servicio o subdirección (directorate) tiene un responsable de evaluación que participa en la red de evaluación. Además participan otros servicios de carácter horizontal como el de valoración de impacto o Impact Assessment. Otras Direcciones Generales funcionan internamente de manera centralizada (por ejemplo: Agricultura, Política Regional, Ayuda Exterior). Existen también redes de evaluación de carácter interno pero inter-sectoriales en las que participan servicios de varias Direcciones Generales. Por ejemplo, la función de evaluación de la Dirección General de Investigación y Desarrollo lidera y coordina una red de evaluación en la que participan múltiples Direcciones Generales (por ejemplo: Sociedad de la Información, Agricultura, Transportes, Medioambiente, Presupuestos).

\section{LA POLÍTICA DE EVALUACIÓN: EN BUSCA DE UN ENFOQUE Y ALCANCE ESTRATÉGICO DE LA EVALUACIÓN}

La decisión sobre qué y cuándo evaluar es descentralizada y no impuesta desde los niveles superiores de la Comisión. El proceso de decisión es más bien de "abajo a arriba" (bottom up). Hasta el 2009, las Direcciones Generales podían decidir realizar una evaluación de manera prácticamente autónoma y por razones de oportunidad, por ejemplo: por necesidades de información, por respuesta a demandas específicas desde el sector privado, etc. La decisión de evaluar puede ser también consecuencia de una obligación legal o de la petición ad hoc de un comisario o de las instituciones de la Unión Europea. En la actualidad el proceso tiene algunas dosis de decisión de "arriba abajo" (top down). La Comisión, a través de la Secretaría General, tiene unas prioridades de temas o políticas a evaluar (género, empleo, competitividad) sobre las que exige un esfuerzo de evaluación especifico si las Direcciones Generales no lo han realizado voluntariamente.

Según la vigente Comunicación sobre evaluación (2007), el objetivo general del sistema de evaluación es orientar su práctica de forma que sus resultados sean más útiles para la toma de decisiones y permitan el desarrollo tanto de las políticas sectoriales como de las prioridades estratégicas de la Comisión (ver Cuadro 2).

Más reciente es la declaración del presidente Barroso sobre la importante contribución que se espera de la evaluación para maximizar la calidad y la eficacia de las propuestas de la Comisión y mostrar que ésta trabaja con responsabilidad y profesionalidad y el diseño de que todas las propuestas significativas de revisión de medidas existentes o de nuevas medidas deberán basarse en una evaluación del marco de la

114 política publica correspondiente (European Commission, 2010). 


\section{CUADRO 2}

\section{Intención de uso de la evaluación como instrumento para la gestión estratégica}

Las políticas comunitarias comprenden una mezcla de iniciativas individuales e instrumentos (gasto, legislación, información, coordinación). El aspecto estratégico de la evaluación radica en la valoración de la relación o influencia de las intervenciones individuales con los objetivos globales de una política a nivel de $A B B$ (Política o Actividad presupuestaria), con los objetivos horizontales (medio ambiente, género), o con los objetivos prioritarios de la Comisión (p.ej. la Estrategia de Lisboa).

Los informes de evaluación deberían incluir preguntas evaluativas, cuando se considere apropiado, que permitan relacionar la intervención evaluada con el marco de política pública en el que se desarrollan, de manera que se ofrezca información sobre: la contribución a los objetivos estratégicos; la coherencia y complementariedad de las intervenciones; el progreso de la Comisión hacia sus objetivos estratégicos... También se deberían incluir de manera creciente preguntas sobre el coste eficacia de y la adecuación de los recursos asignados a la intervención para que los resultados de la evaluación puedan utilizarse para justificar las modificaciones de créditos y el arbitraje entre iniciativas que compiten por recursos presupuestarios. Esta información debería ser útil para las Direcciones Generales implicadas y para los servicios centrales de la Comisión y deberían incorporarse a los procesos de decisión y de presentación de información anual.

La relevancia de ciertos objetivos (Estrategia de Lisboa) o temas horizontales (desarrollo sostenible, genero, etc...) exige que se realicen esfuerzos de evaluaciones con un alcance intersectorial que permita mejorar la coherencia, complementariedad e impacto de las distintas intervenciones de la Comisión que persiguen un mismo fin.

Fuente: European Commission, 2007.

Para avanzar en la orientación estratégica de la evaluación, la Comisión Europea viene trabajando desde 2007 en una serie de medidas relacionadas con el diseño de las evaluaciones, la comunicación e incorporación de sus resultados en los foros y procesos de decisión. Por ejemplo:

a) Asegurar que las evaluaciones se diseñan de manera que faciliten la producción de evidencias e información relevantes y apropiadas para satisfacer las necesidades estratégicas de información de la Comisión Europea. Algunas medidas concretas dentro de estas líneas de mejora son:

- la ampliación del ámbito de aplicación a toda clase de iniciativas, ya sean con gasto o sin gasto (legislación, información, coordinación, etc.).

- la mejora del enfoque de las evaluaciones con la introducción de criterios y cuestiones sobre el valor o la utilidad añadida por las iniciativas evaluadas a las políticas sectoriales y prioridades de las Comisión.

- el ajuste y sincronización de los planes de evaluación a los tiempos de la preparación y aprobación de la legislación y la toma de decisiones en general. 
b) Asegurar que los resultados de la evaluación se comunican y difunden de manera adecuada: mejorando la adaptación y comunicación de los informes de evaluación a los órganos decisorios y a los grupos de interés; renovando los informes anuales y los sistemas de seguimiento de la evaluación (por ejemplo: Evaluation Information Management System; European Innovation Monitoring System); mejorando los documentos presupuestarios y de planificación; reforzando el seguimiento de las recomendaciones de las evaluaciones y los correspondientes planes de acción (por ejemplo: las Fiches Contradictoires).

c) Conseguir una integración eficaz de la evaluación en la fase de la planificación y de elaboración del presupuesto. La evaluación está formalmente integrada en el ciclo de Planificación y Programación Estratégica (SPP) y de gestión de resultados $(A B M)$. Ahora se trata de mejorar los procesos de coordinación y debate entre los servicios involucrados; rediseñar los sistemas y los documentos soporte de información de manera que los resultados de la evaluación se presenten clara y explícitamente y se utilicen eficazmente en los debates de planificación, consulta en procesos de decisión sobre los objetivos políticos y preparación del presupuesto.

Cada año se introducen mejoras en los documentos que incorporan la evaluación en el ciclo de planificación y gestión estratégica, por ejemplo en: los documentos de Política Estratégica Anual (Annual Policy Stratetgy - APS); las memorias presupuestarias o AS (Activity Statements); los Planes Anuales de Gestión (Annual Management Plans - APM); los Planes de Evaluación, etc., para conseguir que la información evaluativa se presente de manera más clara y convincente como evidencias o soportes de decisión.

Desde el 2009 se está reforzando la línea de evaluación de las medidas legislativas (regulaciones del Consejo de Ministros y del Parlamento Europeo). El esfuerzo de conexión entre la evaluación ex ante (Impact Assessment) y la evaluación (continua o ex post) es claro y expreso. Los informes de evaluación de los efectos reales de una medida legislativa tienen que hacer referencia a los efectos previstos en el IA de la misma medida. Y viceversa, la valoración de los impactos estimados de una medida legislativa deben de basarse en las evaluaciones ex post realizadas sobre el marco normativo en cuestión o en fase de modificación. Aunque es pronto para valorarlo, esta nueva línea de esfuerzo puede ir en detrimento del esfuerzo que la unidad de evaluación de la Dirección General de Presupuestos venía realizando con la evaluación de programas de gasto. El acercamiento de la evaluación y el IA, ambos ahora bajo la SG, podría significar un alejamiento de la evaluación del proceso presupuestario. Sin embargo, por el momento no ha sido así y en el 2010 la unidad de evaluación de la Secretaría General sigue participando en las audiencias presupuestarias (Budget Hearings) entre la Dirección General de Presupuestos y las de116 más Direcciones Generales. 
Actualmente la SG tiene seleccionadas unas áreas prioritarias de evaluación con el fin de proporcionar una orientación y estilo de arriba a abajo (top-down) en la política de evaluación en la UE más acorde con los intereses globales comunitarios (por ejemplo: competitividad, medio ambiente, género, y algunos temas horizontales, como puede ser el Método de Abierto de Coordinación).

\section{ANÁLISIS DEL SISTEMA DE EVALUACIÓN DE LA UE CON PARTICULAR REFERENCIA AL USO “PRÁCTICO" DE LA INFORMACIÓN EVALUATIVA}

En base a la información presentada en las secciones anteriores y los datos que se presentan en ésta y en las siguientes secciones, se puede decir que hay argumentos y evidencias suficientes como para aceptar que, aunque con ciertas limitaciones, el sistema de evaluación de la Unión Europea está bien arraigado y se encuentra en una fase de desarrollo orientada a mejorar la calidad y uso de sus resultados, entendido este uso tanto en el marco de la gestión como en el de gobernanza. La validez de esta hipótesis se analiza siguiendo un marco analítico propuesto por Leeuw y Furubo (2008) sobre las condiciones que deben cumplirse para aceptar la existencia de un "sistema" de evaluación en una Administración. Se acepta que este está arraigado y en funcionamiento cuando la actividad evaluadora se realiza: con una perspectiva o identidad cultural y cognitiva comúnmente aceptada; bajo un marco institucional-organizativo coherente (y con capacidad de aprendizaje); de manera permanente y regular; y con intención de uso de los resultados de la evaluación.

En primer lugar, la perspectiva de evaluación es homogénea en toda la Comisión. Existen unas normas, estándares, guías y técnicas de evaluación, etc., comunes a todas las Direcciones Generales, en las que quedan reflejados el significado comúnmente aceptado de la evaluación, la identidad y valores de la actividad evaluadora y su fin último. Los marcos de evaluación sectoriales o especiales de algunas Direcciones Generales son coherentes con el marco común básico. La aproximación metodológica a la evaluación es ampliamente aceptada y se desarrolla de manera participativa y continua (adaptación de standards y guías, examen de calidad, etc.). Las valoraciones de impacto $(I A)$ utilizan metodologías análogas a las de la evaluación. La evaluación esta bien diferenciada de la auditoria y de otros sistemas de inspección y control existentes en la Comisión.

Segundo, como se vio en la sección 3, la evaluación descansa en una organización, consolidada con un marco legal y unos procedimientos claramente diseñados, y funciona de manera descentralizada bajo la coordinación y orientación de la Secretaría General a través de la red de "Funciones de Evaluación” y de unidades de IA de las Direcciones Generales. El sistema de evaluación dispone de mecanismos de aprendizaje organizacional y programático. La red de evaluación funciona como un foro de debate e intercambio de información y conocimiento para mejorar el sistema de evaluación. De sus reuniones surge la creación de grupos de trabajo y seminarios orien- 
tados al desarrollo de procedimientos y técnicas de evaluación. Existen mecanismos regulares de valoración de la calidad de las evaluaciones, y estudios, internos y externos, orientados a introducir mejoras en el sistema de evaluación.

Tercero, el sistema de evaluación de la UE produce y solicita información evaluativa de manera permanente y regular. Las Direcciones Generales, por un lado, producen o subcontratan la realización de actividades de evaluación, y por otro lado, demandan información evaluativa. El colegio de Comisarios y las demás instituciones comunitarias también solicitan información evaluativa (ver Cuadro 1). La producción de evaluaciones es continua y creciente. Desde el 2002 hasta el 2008 se produjeron unas 1200 evaluaciones, unas doscientas al año por término medio (European Commission, 2008a). Todo ello sin contar las numerosas evaluaciones realizadas por los Estados miembros por ejemplo en el dominio de las políticas estructurales. Las evaluaciones tienen una duración media de unos 10 meses y un coste medio aproximado de unos 200.000 euros (European Commission, 2007b).

La última y quizás más importante característica de un sistema de evaluación es la intención de uso de sus resultados. En la UE es obligatorio por ley utilizar los resultados de la evaluación, de hecho aunque de manera limitada la información evaluativa se utiliza, y hay una explícita y renovada declaración de intenciones sobre la orientación practica del esfuerzo de evaluación (European Commission, 2007a y 2010). Los resultados de la evaluación se utilizan como instrumentos de programación y gestión a nivel operativo de las políticas sectoriales. Hasta ahora, su utilidad no se ha considerado relevante para las decisiones estratégicas o presupuestarias (EPEC, 2005) (ver Cuadro 3).

\title{
CUADRO 3
}

\section{Estudio sobre el uso de la evaluación}

\begin{abstract}
... El uso más visible y explícito es a nivel operativo, esto es, en y entre diferentes generaciones de programas de gasto. La mayor parte de las evidencias de uso están relacionadas con el reajuste de la implantación de programas o políticas que están en marcha y en la preparación de sus nuevas fases. El uso de la evaluación como input para el establecimiento de prioridades de políticas públicas es significativamente menor.

$\cdots$

También se han identificado otros usos importantes de la evaluación tales como: la gestión y seguimiento de la Agencias; la formulación de directivas; las negociaciones y preparación de acuerdos con terceros países; etc.

... no existen evidencias de que la asignación de créditos presupuestarios se realice en función de los niveles de eficiencia. En este estudio se muestra que la asignación del presupuesto se realiza de acuerdo con criterios políticos y con relativamente poca información sobre la evaluación. No obstante hay indicios de reasignaciones internas y ganancias de eficiencia dentro de cada intervención gracias a las evaluaciones realizadas (cambios en los criterios de elegibilidad, identificación de ineficiencias operativas).
\end{abstract}

118 Fuente: EPEC, 2005. 
Estos resultados coinciden con los de estudios posteriores en los que se estima que una gran mayoría de las evaluaciones ex ante (IA) no consiguen proporcionar información relevante para el desarrollo de las políticas comunitarias, a nivel estratégico o para temas multisectoriales (European Commission, 2008c).

No obstante, existen algunas excepciones que muestran un cierto uso de información evaluativa a nivel estratégico. Tal es el caso, por ejemplo, de un meta-estudio de las evaluaciones y revisiones de 17 políticas europeas que representaban un $94 \%$ del presupuesto Europeo (Eureval y Rambol, 2008). En este estudio se ofreció una información de síntesis sobre la relevancia, eficiencia, eficacia y sostenibilidad de las iniciativas europeas llevadas a cabo bajo las Perspectivas Financieras de 2000-2006 y las lecciones aprendidas para revisar el gasto de la UE. También se han identificado algunos de $I A$ de utilidad estratégica, presentados acompañando propuestas de medidas de alto perfil político, como por ejemplo la Directiva de Europea de "Aire Limpio" que han sido objeto de intensos debates a máximo nivel de la Comisión (Robertson, 2008).

Así mismo existen casos en los que la evaluación se ha utilizado como instrumento para la reasignación de recursos. A modo de ejemplo de buena práctica, en los dos cuadros siguientes se muestra una parte de la ficha presupuestaria del programa 17.04 (Seguridad Alimentaria y Salud Animal y Vegetal) que la Dirección General de Salud y Consumo presentó en el Proyecto de Borrador Presupuestario (PDB) del 2009, justificando una reasignación de recursos de acciones dirigidas a la erradicación de enfermedades y sus consecuencias (por ejemplo: costes por el sacrificio de ganado infectado) a otras dirigidas a la prevención (por ejemplo: vigilancia y vacunaciones), apoyándose en las conclusiones de una evaluación (ver Cuadros 4 y 5 ).

\section{CUADRO 4}

Ficha Presupuestaria de la Actividad 17.04

Seguridad Alimentaria, Salud Animal y Vegetal

(Anteproyecto de presupuesto 2009)

\begin{tabular}{|l|c|c|}
\hline OBJETIVO ESPECífICO & \multicolumn{2}{|c|}{$\begin{array}{r}\text { Garantizar la seguridad y buen estado de los alimentos } \\
\text { y mantener un alto nivel de salud animal }\end{array}$} \\
\hline $\begin{array}{c}\text { Indicadores de } \\
\text { resultados }\end{array}$ & Últimos resultados conocidos & Metas \\
\hline Reducción de casos de BSE & $2007 / 2008:-42 \%$ & $2009 / 2010:-20 \%$ \\
\hline $\begin{array}{l}N .^{\circ} \text { de casos de salmonela } \\
\text { en humanos }\end{array}$ & $2007: 152.145$ & $2009 / 2010:-10 \%$ \\
\hline Casos de "Blue tongue" & $\begin{array}{l}2007: 50.000 \\
2008: 25.000\end{array}$ & $2009-2010:$ menos de 10.000 \\
\hline
\end{tabular}




\begin{tabular}{|c|c|c|c|c|c|c|c|}
\hline \multicolumn{8}{|c|}{ Principales Resultados (enclave de outputs) de la política al 2010} \\
\hline \multicolumn{8}{|c|}{ Desarrollo de la eco-etiqueta (foot-print), revisión de la legislación medico-alimentaria } \\
\hline \multirow{2}{*}{$\begin{array}{l}\text { Principales resultados } \\
\text { relacionados con el gasto }\end{array}$} & \multirow{2}{*}{$\begin{array}{c}\text { Línea } \\
\text { Presupuestaria } \\
2009\end{array}$} & \multicolumn{2}{|c|}{2008} & \multicolumn{2}{|c|}{2009} & \multicolumn{2}{|c|}{2010} \\
\hline & & $\begin{array}{l}\text { Outputs } \\
\left(\mathrm{n}^{\circ}{ }^{\circ}\right)\end{array}$ & $\begin{array}{c}\text { Mill } \\
\epsilon\end{array}$ & $\begin{array}{l}\text { Outputs } \\
\left(\mathrm{n} .^{\circ}\right)\end{array}$ & $\underset{€}{\operatorname{Mill}}$ & $\begin{array}{l}\text { Outputs } \\
\left(\mathrm{n}^{\circ}{ }^{\circ}\right)\end{array}$ & $\underset{\epsilon}{\text { Mill }}$ \\
\hline $\begin{array}{l}\text { Programas de Vigilancia y } \\
\text { erradicación de TSE, BSE, } \\
\text { y Blue Tongue }\end{array}$ & \multirow{3}{*}{$\begin{array}{l}1704 \\
0101\end{array}$} & 40 & 76 & 51 & 140 & 52 & 170 \\
\hline $\begin{array}{l}\text { Programas de vigilancia } \\
\text { de salmonela }\end{array}$ & & 39 & 29 & 24 & 20 & 24 & 40 \\
\hline $\begin{array}{l}\text { Adquisición de vacunas de } \\
\text { la enfermedad de "Foot y } \\
\text { mouth" ( } \text {. }^{\circ} \text { de dosis) }\end{array}$ & & & 0 & 30 Mill. & 7 & 30 Mill. & 7 \\
\hline Total & & \multicolumn{2}{|l|}{105} & \multicolumn{2}{|l|}{167} & \multicolumn{2}{|l|}{217} \\
\hline
\end{tabular}

Fuente: UE Preliminary Draft Budget 2009.

La primera parte de la ficha (Cuadro 4) ofrece información cuantificada sobre objetivos y resultados en términos principalmente de outputs (algunas en términos de recursos) propios de un sistema de seguimiento. La segunda (Cuadro 5) ofrece una justificación o explicación de la variación de resultados y metas utilizando la información obtenida con la evaluación de la Política Comunitaria de Salud Animal tenida en cuenta por la Comisión para poner un mayor énfasis en medidas preventivas, vigilancia, seguimiento e investigación.

\section{CUADRO 5}

Selección del texto explicativo de la Act. 17.04

\section{Justificación de la variación de los créditos presupuestarios}

... se espera que la tendencia decreciente del número de casos de BSE (vacas locas) continúe y ocasione una reducción de los costes de erradicación y una menor necesidad de apoyo a los Estados Miembros para recoger el ganado infectado (en 2010). Esta reducciones serán más que compensadas por los aumentos en los casos de Bluetongue...

\section{Principales resultados de la evaluación}

...La evaluación CHP - Community Health Policy (1995-2004) apunta... que la CHP ha tenido un éxito creciente en relación con los objetivos que persigue - la reducción en el tiempo de la ocurrencia de un numero significativo de enfermedades animales.......

Como resultado (de la evaluación), la Comisión. tiene como objetivo poner un mayor énfasis en medidas preventivas, vigilancia, seguimiento e investigación (como se explica con los aumentos de ciertos outputs)

\section{Principales logros}

Los problemas de salud animal y seguridad alimentaria se han resuelto con eficacia en 2008. No se dieron casos aparición de brotes significativos de enfermedades serias como la fiebre porcina o "foot and mouth"... Dos países (Alemania y Austria) anunciaron la desaparición de la rabia como resultado de programas de erradicación cofinanciados por la EU... 
Este ejemplo o caso real muestra como la información evaluativa se ha llegado a utilizar de manera coherente y relevante en el proceso presupuestario. Sin embargo, según estudios internos de la Dirección General de Presupuestos sobre la presentación de información evaluativa en una muestra de unas 60 memorias o fichas presupuestarias (Activity Statements) que representaban el 90\% del presupuesto, se necesita realizar importantes mejoras en estas memorias, particularmente en las secciones dedicadas a justificar los créditos solicitados con el uso de los resultados de la evaluación (European Commission, 2008d).

Los estudios mencionados y el caso presentado muestran la posibilidad de uso de la evaluación en gestión y presupuestación por resultados. Pero el avance en este sentido exige la superación de muchas limitaciones aún existentes. Según informes y estudios realizados recientemente en la UE (European Parliament, 2007; ECORYS, 2008) se ha corroborado la dificultad de avanzar con la práctica de la gestión por resultados y la evaluación en la línea de su uso directo e instrumental. A pesar de reconocerse avances significativos, todavía quedan problemas pendientes de resolver e importantes desafíos por confrontar. Por ejemplo:

- En cuanto a la calidad de la información sobre resultados: a) aunque esta va mejorando, en general no alcanzan el nivel de relevancia necesario para ser utilizada en la toma de decisiones; b) Se necesita definir objetivos y seleccionar indicadores de más calidad y relevancia para los decidores a nivel estratégico; c) la información proporcionada sobre la lógica de intervención de las iniciativas europeas es débil y escasa.

- En relación al uso de la información sobre resultados: a) en los últimos años la información evaluativa presentada en los documentos manejados en el proceso de toma de decisiones (APS, AMP, AS) ha mejorado, pero es aún insuficiente y su relación con asignación de recursos es muy débil; b) la información presentada en diversos planes estratégicos (los “Objetivos Estratégicos” de la Comisión; las Perspectivas Financieras; y los Programas Marco y Planes Estratégicos de cada Dirección General) no es todo lo coherente que fuera deseable; c) el nivel de agregación de la información presentada en los documentos de planificación, presupuesto y gestión y el de la información proporcionada por las evaluaciones son diferentes y no permiten un enlace e interpretación clara entre ellos de manera que se facilite su uso en los procesos de decisión; d) muchos programas de actuación son marcos plurianuales (2007-2013) con efectos a largo plazo y la asignación de recursos, anual y a medio y largo plazo, está consolidada por ley, por las perspectivas financieras o por los programas marco. La evaluación puede ser más relevante para las decisiones estratégicas, pero no tanto en los procesos de programación y presupuestación anual, sino al tomar decisiones a medio y largo plazo, por ejemplo en el momento de debatir los programas marco y las Perspectivas Financieras; e) la información evaluativa y lecciones apren- 
didas incorporadas en los informes anuales de actividades al final de año son débiles.

- En cuanto al aspecto cultural y estilos de decisión y trabajo: existe el riesgo de burocratización del ciclo de gestión y presupuestacion por resultados. Aunque ya se dispone de un buen stock de objetivos, indicadores e informes de evaluación, aun se necesita una mayor aceptación de estilos de decisión y de cultura de gestión por resultados: a) la información por resultados no se usa de manera regular para la asignación de recursos; b) no hay motivación para identificar ahorros y acciones eficientes, ni para coordinarse de manera sistemática con departamentos con intereses relacionados; c) cada año se incorporan nuevas prioridades pero no se identifican iniciativas candidatas a reducir su dimensión.

Algunos de los obstáculos y desafíos mencionados podrían superarse con un mayor esfuerzo en la implantación del modelo de gestión por resultados. Pero el problema principal es la gran limitación del modelo de gestión por resultados y el correspondiente rol instrumental (dentro del aspecto técnico-organizacional) de la evaluación para responder a los retos propios de contextos de complejidad e incertidumbre como los que enmarcan las políticas europeas.

\section{MÁS ALLÁ DEL USO TÉCNICO-ORGANIZACIONAL DE LAS ACTIVIDADES DE EVALUACIÓN}

El uso limitado de la información evaluativa en la UE para apoyar las decisiones estratégicas (ver cuadro 3) puede explicarse en la insuficiencia de una evaluación enfocada a lo meramente técnico organizacional y puesta principalmente al servicio de un modelo clásico de gestión por resultados que no se corresponde con la realidad de los procesos de decisión europeos ni es coherente con el contexto. La evaluación y el seguimiento de los resultados de las iniciativas de la UE basados en criterios de valor (pertinencia, eficiencia, eficacia, etc.) y en lógicas de causalidad lineal (input-outputoutcome) son insuficientes para captar, y de hecho normalmente no proporcionan, una información relevante para la identificación de prioridades y la asignación de recursos.

El contexto en el que se desarrollan las políticas comunitarias es de gran complejidad. El tipo de gestión que lleva a cabo la Comisión Europea es principalmente indirecta, con autoridad y responsabilidades descentralizadas o compartidas y no siempre bien definidas y distribuidas entre diversas instituciones y niveles de gobierno. Los procesos de decisión y ejecución de las políticas europeas se realizan bajo la influencia de no pocos actores con intereses y valores diversos. La dimensión y naturaleza de los problemas y las lógicas de intervención son confusas y cambiantes. A menudo los objetivos son genéricos o premeditadamente ambiguos y abiertos a la

122 negociación. Lo más relevante (outcomes, impactos) suele ser de muy difícil medida 
y atribución. Muchas políticas europeas son funcionalmente interdependientes (por ejemplo: medio ambiente y agricultura, sanidad y mercado único, empleo y desarroIlo regional).

Desde hace unas cuatro décadas, diversas escuelas clásicas de evaluación se han centrado en el estudio de las dificultades contextuales que influyen en el uso de la información evaluativa, tratando de darles respuesta con distintos modelos o aproximaciones a la evaluación: "enlightening model” (Weiss, 1973, 1983); "policy shaping” (Cronbach, 1980); "responsive-participatory evaluation” (Stake, 1980; Guba y Lincoln, 1989); Teory Based Evaluation (Weiss, 1997) "Utilization Focused evaluation" (Patton, 1997). Sería interminable la lista de los estudios realizados sobre el uso de la evaluación en los últimos años (Feinstein y Puetz, 2000; Pawson, 2002; Stame, 2004; Rist y Stame 2006; Feinstein, 2007; Stame, 2008; Stern, 2009).

En la Comisión, el concepto de "utilidad" de la evaluación a menudo se plantea y debate en términos estrechos, entendida como la aplicación mecánica y directa de las recomendaciones propuestas por un informe de evaluación para realizar cambios inmediatos que resuelvan problemas concretos de gestión o programación. Esta percepción de la utilidad mecánica y automática está muy lejos de la realidad de los largos y complicados procesos de decisión y desarrollo de las políticas comunitarias. El abanico de posibilidades para reconocer y mostrar la utilidad de la evaluación es amplio, por ejemplo: concienciar a los actores de las políticas públicas, iniciar cambios en la percepción de los problemas, enriquecer el debate y reorientar la agenda política, reducir incertidumbre sobre la lógica de intervención, etc.

Por otro lado, son muchos los factores que influyen en el uso de la evaluación: la calidad de la evaluación, los métodos e instrumentos elegidos, la estrategia de comunicación de los resultados de la evaluación, o la consideración de la utilidad no ya de cada trabajo de evaluación sino por el valor añadido de una serie de evaluaciones o un flujo de información evaluativa de origen diverso (Vedung, 1993; Rist y Stame, 2006).

En este artículo se destaca la gran importancia que, para el uso de la evaluación, tiene la coherencia entre el modelo de dirección, la evaluación y el contexto en el que se quiere aplicar sus resultados. En esencia se trata de identificar qué modelo de decisión representa mejor la realidad de los procesos de preparación y desarrollo de las políticas comunitarias y qué aproximación a la evaluación sería más adecuado para añadir valor en este proceso. El contexto de complejidad e incertidumbre de las políticas (reformas) europeas exige un cambio (innovación) de tipo más adaptativo y que programado (Berman, 1980) que tenga en cuenta la conveniencia y capacidad de participación, consulta y coordinación, que acepte la necesidad de negociación abierta, la acción conjunta, etc. en las distintas fases del ciclo de las políticas públicas. 
La iniciativa de Gobernanza Europea con sus principios, presentados en la sección II, se justifica apelando no sólo al déficit democrático en la UE, sino además al déficit de capacidad de coordinación y coherencia (Metcalfe, 2004) para llevar a cabo con eficacia la dirección europea. Los principios de Gobernanza Europea se orientan a cubrir estos déficits teniendo en cuenta la realidad desestructurada del contexto de las políticas comunitarias. Se habla también de un "déficit de evaluación" (Stame, 2004) en la UE, relacionado con la carencia o débil desarrollo de una aproximación a la evaluación que responda a los retos del contexto, como la que se sugiere con la Evaluación Basada en la Teoría-TBE (Weiss, 1997). Esta aproximación reconoce la importancia de la lógica de la intervención, más aún cuando ésta se basa en las interacciones entre niveles de gobierno y múltiples actores o grupos de interés (Stame, 2008).

La transparencia, la comunicación, la interacción, el trabajo en red, etc., son ampliamente reconocidas, tanto en el sector público como en el privado, como factores claves de la creatividad, innovación y flexibilidad necesaria para responder a la incertidumbre propia de los contextos de complejos o desestructurados (Berman, 1980; Metcalfe, 1993; Lorenzo, Kawaleck y Ramdani, 2009). Por el contrario, la aproximación estandarizada de la gestión por resultados, basadas en lógicas secuenciales y cerradas, desincentiva la adaptabilidad de las organizaciones (Perrin, 2002). Pawson and Tilley, citados por Stame (2004) proponen una "evaluación realista" basada en una "teoría generativa de la causalidad" o una lógica interactiva y abierta. La innovación o el cambio no lo produce el programa sino las reacciones de los actores y grupos interesados en su implantación. La evaluación en el marco de la gobernanza considera también la participación e influencia de los múltiples actores en la fase de diseño de la intervención publica. Por tanto, por coherencia con un contexto de incertidumbre, la utilidad y relevancia estratégica (innovadora) de la evaluación se ubica en el marco de la iniciativa de gobernanza.

Para poner en práctica la Gobernanza Europea y sus principios se han ido aplicando nuevos instrumentos de políticas, tales como los procesos de co-regulación, diferentes formas de partenariado, el funcionamiento en red, el Método Abierto de Coordinación (OMC, por sus siglas en inglés). Todo ello exige un tipo de aproximación especial a la evaluación: basada en un modelo (por ejemplo: el TBE) que permita captar las relaciones causales de la Gobernanza; con distintos objetos de evaluación (los nuevos instrumentos); enfoque (estratégico) y metodología (por ejemplo: evaluación participativa, análisis de redes) (Stame, 2008; Stern, 2009, Aguilar y Bustelo, en este número).

En los últimos años, la Comisión ha utilizado de manera creciente la evaluación como instrumento para la gobernanza de las políticas comunitarias. Son diversas las evaluaciones realizadas por la Comisión que se han centrado en el examen de la aplicación de los principios de gobernanza europea y al uso de sus instrumentos

124 (por ejemplo: participación, coherencia, partenariados, Open Method Coordination, 
network building, etc.). Son múltiples las evaluaciones realizadas por los Estados miembros sobre el funcionamiento del partenariado en el marco de los Fondos Estructurales. Además, en los informes de evaluación se dedican secciones o apartados al grado de participación en las intervenciones evaluadas y su coherencia con otras políticas. A continuación se ilustra la utilidad de la evaluación, valorada en referencia al cumplimiento de los principios gobernanza y la eficacia de los instrumentos de gobernanza.

En los siguientes cuadros, 6 y 7, se presentan ejemplos y casos de buenas prácticas en los que la relación entre la evaluación y dos instrumentos de gobernanza es preeminente y en los que se muestra la relevancia de los resultados de la evaluación para mejorar los instrumentos y las iniciativas comunitarias correspondientes. En una evaluación realizada en 2005 por consultores externos para la Dirección General de Información y Sociedad de la Comisión Europea, se identificaron una serie de importantes dificultades para la aplicación del Open Method Coordination (OMC) en la estrategia de "e-Europe" orientada a mejorar la accesibilidad y conexión en línea entre individuos, hogares, escuelas, empresas y la Administración en distintos sectores (transporte educación, salud, etc.). La aplicación de este método de coordinación se encontró con retos de implantación tales como: la necesidad de ajustar y adaptar constantemente las metas y compromisos (benchmarks) dado los continuos cambios contextuales; la diversidad de marcos institucionales y características de los mercados en el terreno de la Sociedad de la Información en cada país; la falta de mecanismos eficaces para el intercambio y difusión de buenas prácticas; y particularmente la débil capacidad de coordinación interna dentro de los propios Estados miembros, con especial mención del caso español, donde "los fuertemente autónomos gobiernos regionales, liderados por distintos partidos políticos, tienen una amplia gama de iniciativas de Sociedad de la Información y no se dispone de suficiente capacidad de coordinación interregional o nacional-regional. Así mismo, en esta evaluación se analizó de manera comparada el alcance del uso del método OMC en otras políticas comunitarias identificando posibles líneas para mejorar este instrumento de gobernanza europea (ver Cuadro 6).

En otro caso, la evaluación del VI Programa Marco de Tecnología en la Sociedad de la Información (FP6 IST), se utiliza el análisis de redes como un instrumento de evaluación. Uno de los objetivos del FP6 IST era la integración de investigadores, expertos, empresarios y otros actores en el Área Europea de Investigación (European Research Area-ERA). Con este programa se está realizando un claro esfuerzo de fomento de redes y actividades de colaboración principalmente entre centros de investigación y conocimiento tecnológico, entre proyectos de I+D, y entre científicos. La colaboración en la investigación se percibe como un medio y como un fin en sí mismo. Se entiende como instrumento de intercambio de información y de innovación que puede alcanzarse facilitando la creación y consolidación de redes autogobernadas. Los resultados de esta evaluación se presentan en forma de cambios en las características de las redes analizadas. 


\section{CUADRO 6}

\section{Evaluación del Método Abierto de Coordinación (Open Method Coordination - OMC)}

Del análisis comparado sobre el funcionamiento del sistema de OMC en diversas políticas comunitarias los consultores sacaron las siguientes conclusiones generales:

- No se ha implantado todavía de manera plena en muchas políticas comunitarias. Se ha avanzado en la concienciación y sensibilidad de los Estados miembros, en clarificación de indicadores y benchmarks y en la promoción de las iniciativas nacionales que ya estaban en curso. Pero, con la excepción de la política de Empleo y Asuntos Sociales, el aprendizaje mutuo y los instrumentos de revisión por el grupo de pares (peer review) apenas han sido desarrollados.

- Parece haber favorecido el desarrollo de una cultura colegiada entre Estados miembros, la Comisión, y los entes regionales, locales y transnacionales, con el fortalecimiento de valores propicios al intercambio de información, solución conjunta de valores, identificación de objetivos comunes, responsabilidad reciproca.

- No ha funcionado en base a la identificación de fracasos y culpables o éxitos y fama ("Name and Shaming or Faming"), sino que más bien ha provocado prácticas de resistencias a proporcionar información o de deformación de información.

Así mismo, la evaluación identifica varias líneas de mejora del OMC:

- Mayor concentración y énfasis en el establecimiento de procesos prácticos y eficaces para la identificación, diseño y acuerdo sobre los objetivos y compromisos estratégicos y substantivos de manera específica para cada EM.

- Implantación de un verdadero proceso de revisión por pares más orientado al aprendizaje mutuo, basado en el apoyo, contextualización y desarrollo pero con cierto espacio para el debate, la lógica, la solución de problemas y las recomendaciones.

- Utilizar indicadores y benchmarks no tanto para identificar culpabilidades o éxitos, sino para facilitar la identificación de dificultades y la definición de tareas al establecer los objetivos, metas y compromisos.

- Involucrar y movilizar una amplia gama de actores entre los representantes sociales, grupos empresariales, sociedad civil, y los gobiernos regionales y locales en el proceso de OMC.

Fuente: Tavistock Institute, Net Effect Ltd., Istituto per la Ricerca Sociale, (2005).

A pesar de estas dos buenas prácticas, aún es posible aprovechar mejor el potencial de la evaluación para el desarrollo de la capacidad de gobernanza. No obstante, varias Direcciones Generales (Empleo, Sociedad de la Información, Investigación y Desarrollo) y la Secretaría General están particularmente interesadas en reforzar y favorecer el desarrollo de la evaluación en esta línea. Como se anticipó en apartados anteriores, una de las prioridades de evaluación de la SG para los próximos años es la valoración del OMC. Es de esperar que los recursos y esfuerzos de evaluación se 126 orienten también en este sentido. Sin duda habrá que evaluarlo. 


\section{CUADRO 7 \\ El análisis de redes como instrumento de evaluación del VI Programa Marco de Tecnología en la Sociedad de la Información (FP6 IST)}

La evaluación de las redes de investigación en el FP6 IST ha proporcionado información sobre la dinámica de cambios en la colaboración que no se podrían haber identificado con otros métodos de evaluación. Por ejemplo:

- Los participantes de las redes del FP6 están más firmemente interconectados que en los anteriores FP. Hay más enlaces, en términos relativos al número de organizaciones participantes. Esta conectividad ofrece posibilidades de acceso a otros grupos que realizan investigaciones complementarias.

- Los proyectos están más estrechamente conectados que en el FP5. Es decir se facilita la cros-fertilización entre distintas actividades de investigación.

- El FP6 está mejor integrado que otros programas de europeos (EUREKA, COST).

- La conexión fluye a través de un mayor número de rutas lo que supone una mayor flexibilidad de la red.

Según el informe de evaluación, las redes del FP6 tienen más posibilidades de:

- Integrar las universidades y las industrias en proyectos de investigación conjunta, "característica que no se da en la mayoría de actividades no financiadas con fondos EU por ser colaboraciones solo inter universitarias o solo entre empresas".

- Conectar científicos de distintas disciplinas.

- Incluir más de 3 EEMM de ERA (European Research Area) en el equipo de un proyecto.

- Incluir propietarios de patentes y organizaciones frecuentemente citadas.

- Incorporar pequeñas y medianas empresas en los equipos de investigación.

Además se identifica que la mayoría de los participantes centran su interés en innovaciones relacionadas con los métodos y formas de trabajo (con una perspectiva interna) más que en la generación (externa) de nuevos productos o servicios, lo que sugiere que una evaluación enfocada a la valoración de outcomes comerciales no hubiera captado el valor añadido intangible de este programa.

Fuente: RAND Europe, 2005.

\section{CONCLUSIONES}

En los últimos años la Comisión Europea ha hecho grandes esfuerzos y ha conseguido significativos avances en la institucionalización y funcionamiento de su sistema de evaluación. Se han mostrado claras evidencias de que hoy día el sistema de evaluación en la UE está bien arraigado (visión conceptual homogénea, normativa y organización consolidada, oferta y demanda continuada) y se encuentra en fase de desarro- 
Ilo para mejorar su calidad y uso. Son diversos los factores mencionados en el texto que han podido influir en este avance, por ejemplo: la obligación legal clara y explícita de evaluar y utilizar sus resultados para asignar recursos; el interés por parte de las instituciones comunitarias y de la alta dirección de la Comisión; la revisión regular del funcionamiento del propio sistema de evaluación y la capacidad de aprendizaje y mejora del sistema.

En la práctica los resultados de la evaluación se utilizan de manera restringida, esencialmente para la toma de decisiones relacionadas con la implantación de programas y su reprogramación operativa, la gestión y seguimiento de las Agencias, y la preparación de acuerdos y negociaciones. Se han podido identificar casos de buenas prácticas de uso real de la evaluación y se ha mostrado la intención política y las líneas de actuación para conseguir una utilidad de mayor relevancia estratégica y más coherente con el contexto europeo. Sin embargo, siguen existiendo importantes limitaciones de evaluabilidad y es muy débil el uso de la información evaluativa a niveles estratégicos. Se ha argumentado que el gran obstáculo para aumentar la utilidad de la evaluación es la debilidad y poca relevancia del modelo de gestión por resultados en los entornos desestructurados.

En este trabajo se ha destacado la gran importancia que, para el uso de la evaluación, tiene la coherencia entre el modelo de evaluación, la naturaleza del proceso de decisión y el contexto en el que se quiere aplicar sus resultados. El contexto de las políticas europeas se caracteriza por la incertidumbre, el pluralismo y la diversidad, exigiendo una aproximación a la dirección Europea de tipo flexible y participativo, que tenga en cuenta la conveniencia y capacidad de coordinación y consulta, que acepte la necesidad de participación abierta, la negociación transparente, y la acción conjunta en todas las fases del ciclo de las políticas europeas. Este planteamiento es acorde a la iniciativa de gobernanza.

La iniciativa de Gobernanza Europea se justifica no sólo apelando al déficit democrático en la UE sino además como respuesta al déficit de capacidad de coordinación y coherencia para llevar a cabo con eficacia la dirección europea. Los principios de Gobernanza Europea se orientan a cubrir estos dos déficits teniendo en cuenta la realidad del contexto de las políticas comunitarias. La transparencia, la comunicación, la interacción, y el trabajo en red, son factores claves de la creatividad, innovación y adaptabilidad necesaria para responder a la incertidumbre.

Dada su coherencia con el contexto de las políticas comunitarias, la Gobernanza parece ser un modelo de dirección apropiado y con clara proyección de futuro (Stern, 2009). Por tanto, el valor estratégico de la evaluación en la UE exige una aproximación en el marco de la iniciativa de gobernanza, con distintos objetos, enfoque y métodos. Este modelo proporciona un gran potencial de desarrollo para la evaluación. Como hemos visto, la Gobernanza Europea se beneficia, en alguna medida, de las 128 posibilidades que ofrece la evaluación y viceversa. 
Todo apunta a que la relevancia del beneficio aportado por la evaluación a la dirección de la UE podría aumentar de manera significativa, especialmente a nivel estratégico, si los recursos y esfuerzos de evaluación de la Comisión se reorientaran a favor de la línea de gobernanza. La práctica de la evaluación bajo este modelo promete una información de gran utilidad potencial para las iniciativas europeas. Esta afirmación queda abierta al debate y la comprobación de su materialización y resultado futuro.

\section{REFERENCIAS BIBLIOGRÁFICAS Y DOCUMENTALES}

Argyris, C. (1982), Reasoning, Learning, and Action. San Francisco: Jossey-Bass Publishers.

Berman, P. (1980), “Thinking about programmed and adaptative implementation”, en H. Ingrand y D. Mann, eds., Why Policies Succed or Fail? Londres: Sage.

Borrás, S. (2009), “La Comisión Europea como intermediario en las redes de políticas públicas”, Gestión y Análisis de Políticas Públicas, Nueva Época, 1: 9-42

Borrás, S. y Jacobsson, K. (2004), "The open method coordination and the new governance patterns in the EU", Journal of the European Public Policy, 11(2): 185208.

Consejo de Ministros de la UE (2008), Results of the meeting of the Budget Committee of the Council of Ministries of the EU, 3 de octubre (no publicado).

Cronbach, J. L. et al. (1980), Toward Reform of Program Evaluation. San Francisco: Jossey-Bass.

Diamond, J. (2003), "Performance Budgeting: Managing the Reform Process", FMI Working Paper, WP/03/33.

ECA (2006), Informe sobre la Evaluación en la Comisión Europea del año 2006. Luxemburgo: European Court of Auditors.

Eureval y Rambol (2008), Meta study on lessons from existing evaluations as an input to the review of $E U$ spending. Bruselas: Evaluation for the European Commission.

Ernst \& Young (2008), Result-Based management at thee Heart of a new public management: An Overview of public policy evaluation in Europe. París: Ernst \& Young. En línea: <http://www2.eycom.ch/publications/items/public_services/2008_public_policy_evaluation/2008_EY_Overview_Public_Policy_Evaluation.pdf $>$ (Consulta: abril 2010).

ECORYS Nederland BV (COWI Consortium) (2008), Study on the state of implementation of Activity Based Management in the European Commission. Informe preparado para la Comisión Europea. Bruselas: Directorate-General for Budget.

European Commission (1996), Evaluation Communications. En línea: <http://europa.eu.int/comm/budget/evaluation/keydocumentsfr.html> (consulta: 7 enero 2010).

European Commission (1999), Communication on Activity Based Budget - ABB. SEC 148/9, Bruselas: European Commission.

European Commission (2000), Evaluation Communications. En línea: <http://europa. eu.int/comm/budget/evaluation/keydocumentsfr.html> (consulta: 7 febrero 2010). 
European Commission (2001a), Communication on Implementing Activity Based Management in the Commission. SEC 1197/687. Bruselas: European Commission.

European Commission (2001b), European Governance: A White Paper. Bruselas: Commission of the European Comunities, COM: 428. En línea: <http://ec.europa.eu/ governance/white_paper/en.pdf>.

European Commission (2001C), "Report of the Working Group «Evaluation and transparency»", en European Governance: $A$ White Paper. Bruselas: Commission of the European Comunities, COM: 428.

European Commission (2002a), Reglamento Financiero de la UE, Regulación del Consejo 1605/2002. Bruselas: European Commission.

European Commission (2002b), Communication from the Commission on Impact Assessment. Bruselas: Commission of the European Comunities, COM: 276.

European Commission (2004), Evaluating EU Activities: A Practical Guide for the Commission Services. Bruselas: European Commission. En línea: <http://europa.eu.int/ comm/budget/evaluation/keydocumentsfr.htm> (Consulta: 7 febrero 2010).

European Commission (2005a), Better Regulations for Growth and Jobs in the European Union. Bruselas: Commission of the European Comunities, COM: 97.

European Commission (2005b), How the e-Europe OMC worked: Implications for the Coordination of Policy under 2010. Bruselas: European Commission. En línea: <http://ec.europa.eu/dgs/information_society/evaluation/data/pdf/studies/2005_omc_Finalrep.pdf> (Consulta: 7 febrero 2010).

European Commission (2007a), Evaluation Communication. Bruselas: European Commission. En línea: <http://europa.eu.int/comm/budget/evaluation/keydocumentsfr.htm> (Consulta: 7 febrero 2010).

European Commission (2007b), Evaluation in the Commission: Reporting on Evaluation Capacity 2006. Documento de trabajo interno. Bruselas: Directorate General Budget.

European Commission (2007c), Using evaluation results in the Activity Statements of the PDB. Internal Study 2007. Bruselas: Directorate General Budget.

European Commission (2008a), Multiannual Evaluation Report. Bruselas: European Commission. En línea: <http://europa.eu.int/comm/budget/evaluation/keydocumentsfr.htm> (Consulta: 7 enero 2010).

European Commission (2008b), Reglamento Financiero 1605/2002 del Consejo de Ministros de la Unión Europea. Bruselas: European Commission. En línea: <http://europa.eu.int/comm/budget/evaluation/keydocumentsfr.htm> (Consulta: 7 febrero 2010).

European Commission (2008C), IAB Report for the year 2008, Commission Staff working document, SEC 55. Bruselas: European Commission. En línea: <http://eurlex.europa.eu/LexUriServ/LexUriServ.do?uri=CELEX:52009SC0055:EN:NOT> (Consulta: 7 febrero 2010).

European Commission (2008d), Incorporating evaluation results in AMPs and Activity Statements.. Documento interno: A01(2008)D 51673. Bruselas: Secretariat General and General Directorate for Budget

European Commission (2009), Evaluation Quality Assessment Framework, Secretary 130 General. Bruselas: European Commission. En línea: <http://ec.europa.eu/dgs/se- 
cretaría t_general/evaluation/docs/how_quality_asses_0206_en.pdf> (Consulta: 7 febrero 2010).

European Commission (2010), Working Methods of the Commission 2010-2014. En línea: <http://myintracomm.ec.europa.eu/NewsPortal/Documents/workingmethods_en.pdf> (Consulta: 7 febrero 2010).

European Parliament (2008), Study on the Administrative reforms of the Commission in relation to $A B B / A B M$. Committee on Budgets, Bruselas: European Parliament.

EPEC (2005), Study on the use of Evaluation results in the Commission. Bruselas: European Policy Evaluation Consortium.

Feinstein, O. y Puetz, D. (2000), "Synthesis and Overview", en O. Feinstein y R. Picciotto, Evaluation and Poverty Reduction. Washington: The World Bank.

Feinstein, O. (2007), "Evaluación pragmática de políticas públicas”, Información Comercial Española, 836: 19-31

Guba, E. G. y Lincoln, Y.S. (1989), Fourth Generation Evaluation. Londres: Sage.

International Monetary Fund (IMF) (2005), Performance Budgeting: linking results and Funding. En línea: <http:/www-int.imf.org/depós/fad/pdrp/conference/perf_budget/index.html> (Consulta: 7 febrero 2010).

Jordana, J., Mota, F. y Noferini, A. (2009), "La política de Cohesión Europea en dos regiones españolas: Configurando las redes de políticas y el capital social”, Gestión y Análisis de Políticas Públicas, Nueva Época, 1: 87-122.

Leeuw, F. y Furubo, J. (2008), “Evaluation Systems”, Evaluation, 14(2): 157-169.

Lorenzo, O., Kawaleck, P. y Ramdani, B. (2009), “The Long Conversation: Learning How to Master Enterprise Systems", California Management Review, 52(1).

Majone, G. (1991), "Cross-National Sources of regulatory policy making in EU and US", Journal of Public Policy, 11(1): 79-106

Metcalfe, L. (1993), "Public Management: From Imitation to Innovation”, Australian Journal of Public Administration, 52(3): 174-189.

Metcalfe, L. (1995), “La Comision Europea como una organizacion-red”, Gestión y Análisis de Políticas Públicas, 4: 25 - 36.

Metcalfe, L. (2001), "Reforming European Governance: Old Problems or New Principles?”, International Review of Administrative Sciences, 67: 415-443.

Metcalfe, L. (2004), "European Policy Management: Future Challenges and the Rol of the Commission", Public Policy and Administration, 19(3): 77-94.

OECD (1996), Budgeting for Results: Perspectives on Public Expenditure Management. París: OECD.

OECD (2002), Overview of Results Focused Management and Budgeting in OECD Member Countries. Paris: OECD.

OECD (2007), Performance Budgeting in OECD Countries. Paris: OECD.

Patton, M. Q. (1997), Utilization Focused Evaluation. Londres: Sage

Pawson, R. (2002), “Evidence Based Policy: The Promise of 'Realist Synthesis'”, Evaluation, 8(3): 340-358.

Perrin, B. (2002), "How to -and How Not to- Evaluate Innovation”, Evaluation, 8(1): 13-28.

Pollitt, C. y Bouckaert, G. (2000), Public Management Reform: A comparative Analysis. Oxford: Oxford University Press. 
RAND Europe (2005), “ERAnets Evaluation of Networks of collaboration among participants in the IST Research and their evolution to collaborations in the ERA". Informe final. Bruselas: DG Information Society.

Radej, B., Golobic, M. y Cernic, M. (2010), "Beyond New Public Management doctrine in policy impact evaluation”, Working paper 1/2010 Slovenian Evaluation Society: 1-25.

Renda, A. (2006), Impact Assessment in the EU: The State of the Art and the Art of the State. Bruselas: Centre for European Policy Studies.

Rieper, O. (1997), “Intergovernmental Evaluation of an EU-Funded Regional Development Program in Denmark", en O. Rieper y J. Toulemonde, eds., Politics and Practices of Intergovernmental Evaluation. New Brunswick: Transaction Publishers.

Robertson, C. (2008), "Impact assessment in the EU”, EIPA Scope: 17-20 En línea: <http://www.inmetro.gov.br/IVWAC/apresentacoes/avaliacao_de_impactos_regulatorios.ppt\#326,1,Impact Assessment in the European Union> (Consulta: mayo 2010).

Robinson, M. (2007), Performance Budgeting - Linking Funding and Results. Palgrave: McMillan.

Rist, R. y Stame, N. (2006), Evaluative Knowledge Management: from Studies to Streams. New Brunswick: Transaction Publishers.

Scharpf, F.W. (2002), Governing in Europe: Effective and Democratic? Oxford: Oxford University Press.

Stake, R. (1980), "Program evaluation, Particularly Responsive evaluation”, en W. B. Dockrell y D. Hamilton, eds., Rethinking Educational Research. Londres: Hodder and Stoughton.

Schick, A. (2001), “Does Budgeting have a future?”, Ponencia presentada en el 22nd Annual Meeting of Senior Budget Officials, Paris 21-22 de mayo.

Stame, N. (2004), "Theory-Based Evaluation and Types of Complexity", Evaluation, $10(1): 58-76$.

Stame, N. (2008), “The European Project, Federalism and Evaluation”, Evaluation, 14(2): 117-139.

Stern, E. (2009), "Evaluation policy in the European Union and its institutions", New Directions for Evaluation, 123: 67-85.

Subirats, J. (2004), “¿Podemos utilizar los instrumentos de la evaluación como palanca de gobierno del sector público?" Ponencia presentada en el IX Congreso Internacional del CLAD sobre Reforma del Estado y de la Administración Pública. Madrid, noviembre.

Summa, H. y Toulemonde, J. (2002), "Evaluation in the European Union: Addressing Complexity and Ambiguity”, en J. E. Furubo, R. Rist y R. Sandhal, eds., International Atlas of Evaluation. New Brunswick: Transaction Publishers.

Tavistock Institute, Net Effect Ltd., Istituto per la Ricerca Sociale (2005), "The Analysis of Impacts of Benchmarking and the eEurope Actions in the Open Method of Co-ordination - How the eEurope OMC worked: Implications for the Co-ordination of Policy under 2010". Informe Final. Bruselas: DG Information Society of the European Commission.

Vedung, E. (1993), “La Utilización de la Evaluación”, Revista de Servicios Sociales y Po132 lítica Social, 30: 69-80. 
Weiss, C. (1973), “Where politics and Evaluation Research meet", Evaluation, 1: 37-45. Weiss, C. (1983), "Ideology, Interest, and Information: The basis for policy decisions", en A.S. Bryk, ed., Stakeholder-Based Evaluation. San Francisco: JosseyBass.

Weiss, C. (1997), "Theory Based Evaluation: Past, Present and Future”, New Directions for Evaluation, 76: 41-55.

Recibido: 3 de marzo de 2010

Aceptado: 30 de marzo de 2010 